\title{
Thank you to the journal's assessors
}

J Med Ethics 2002;28:55

We wish to thank the assessors, listed below, who have helped on recent editions of the journal. Their advice, guidance, and support is greatly appreciated.

Dr Gwen Adshead UK Professor Alastair Ager UK Dr Priscilla Alderson UK Professor Judy Allsop UK Professor Brenda Almond UK Dr Marianne Arndt UK Assistant Professor D G Arnold USA Dr A Asai JAPAN Dr Richard Ashcroft UK Dr E Aydin TURKEY Professor Tom Baldwin UK Professor R W Beard UK Dr Peter Beck UK Dr Piers Benn UK Ms R Bennett UK Dr A C Berry UK Dr Hazel Biggs UK Professor A C Bird UK Professor Bela Blasszauer HUNGARY

Dr Sara Booth UK

Professor Nick Bosanquet UK Professor Fouad Boustany

LEBANON

The Revd Dr Kenneth M Dr Peter Bradley NORWAY

Dr Ann Bradshaw UK

Dr Bob Brecher UK

Dr Mark Brennan UK

Dr Edwina Brown UK

Mr R E Bryden UK

Professor Peter Byrne UK

Professor Alastair Campbell UK

Professor David Carr UK

Professor Thomas Cavanaugh USA

Professor Ian Chalmers UK

Dr Timothy Chappell UK

Dr Angus Clarke UK

Dr Graham Clayden UK

Dr Adrian Cook UK

Professor Roger Cooter UK

Dr Oonagh Corrigan UK

The Revd Peter Cowell UK

Professor Cheryl Cox McPherson GRENADA

Dr Alan Cribb UK

Dr Marina Cuttini ITALY

Dr John Dawson UK

Dr Lach de Crespigny AUSTRALIA

Dr Paquita de Zulueta UK

Professor Donna Dickenson UK

Ms Kate Diesfeld NEW ZEALAND

Ms Alison Dines UK

Dr Habzade Dogan TURKEY

Dr Wybo Dondorp

THE NETHERLANDS

Professor Robin S Downie UK

Dr Derek Doyle UK

Dr Heather Draper UK

Professor Gordon R Dunstan UK

Professor Gerald Dworkin USA

Dr O Dyar UK

Dr S Edwards UK

Dr Sarah Edwards UK

Dr T Ellershaw UK

Dr Charles Erin UK
Professor E Ernst UK

Dr Martyn Evans UK

Professor Don Evans

NEW ZEALAND

Professor Gavin Fairbairn UK

Dr Dominic H R Faux UK

The Revd Nick Fennemore UK AUSTRALIA

Professor R Fitzpatrick UK

Dr David B Fletcher USA

Professor Duncan Forrester UK

Dr Claire Foster UK

Dr Ann Furedi UK

Professor D J Galton UK

Ms Tina Garanis-Papadotos GREECE

Dr Alex Gatherer UK

Professor B G Gazzard UK

Dr James Gilbert UK

Professor Robin Gill UK

Dr Lynne Gillam AUSTRALIA

Professor Raanan Gillon UK

Professor MUDr Ivan Gladkij CSc CZECH REPUBLIC

Dr MUDr Jozef Glasa

SLOVAC REPUBLIC

The Reved Malcolm Goldsmith UK

Associate Professor Dr Sefik

Gorkey TURKEY

Dr Roger Goss UK

Dr David Greaves UK

Professor Sir John Grimley Evans

UK

Dr Marilys Guillemin AUSTRALIA

Ms Janet Hadley UK

Dr Angela Hall UK

Dr Cathy Hammerman ISRAEL

Ms Jean Harbison UK

Mr David Harris UK

Professor Helen Haste UK

The Revd Peter Haughton UK

Dr Matti Hayry FINLAND

Mr Adam Hedgecoe UK

Dr Sirkku Hellsten TANZANIA

Dr T M Helme UK

PD Dr Wolfram Henn GERMANY

Dr Andrew Herxheimer UK

Dr Fiona Hicks UK

Professor Roger Higgs UK

Dr Mark Hilberman USA

Mr J S Hillman UK

Professorr R D Hinshelwood UK

Sir Raymond Hoffenberg UK

Dr Soren Holm UK

Professor J Stuart Horner UK

Dr C Howard UK

Dr Jonathan Hughes UK

Dr Julian C Hughes UK

Dr Jeremiah Hurley CANADA

Dr Trevor Hussey UK

Dr Francoise Hutton UK

Dr Richard Huxtable UK

Ms Jennifer Jackson UK

Professor W B Jennett CBE UK

Professor A G Johnson UK

Dr Jane Kaye UK
Dr John Keown UK

Mr R H J Kerr-Wilson UK

Dr Ian Kerridge UK

Dr Jane Kidd UK

Mr Simon Kirchin UK

Mr C G Koffman UK

Professor Dr Michael Kottow CHILE

Professor O I Kubar RUSSIA

Professor Arthur Kuflik USA

Dr Helga Kuhse AUSTRALIA

Dr David Lamb UK

Dr Ellie Lee UK

Dr Gordon Lennox UK

Dr Kathleen Liddell UK

Professor R J Lilford UK

Dr Richard Lindley UK

Professor Brian Livesley UK

Dr David Lloyd UK

Dr Nick Longford UK

Dr Steven Luttrell UK

Sir A W Macara UK

Professor Darryl Macer JAPAN

Dr Jane Macnaughton UK

Dr K D MacRae UK

Mr David Malyon UK

Dr Robert Maxwell UK

Dr A McCall Smith UK

Dr Tony McGleenan UK

Miss Jean McHale UK

Dr Rosemary McIntyre UK

Dr Shirley McIver UK

Ms Judy McKimm UK

Mr C J McKnight UK

Dr Hugh McLachlan UK

Professor Sheila McLean UK

Professor C McManus UK

Dr John McMillan UK

Dr John McPhee AUSTRALIA

Dr Chris Megone UK

Professor V L Melnick USA

Dr Jose Miola UK

Mr Derek Morgan UK

Professor David B Morton UK

Dr Kim Mulholland AUSTRALIA

Professor Timothy F Murphy USA

Dr Mark Nelson UK

Dr Christopher Newdick UK

Dr Justin Oakley AUSTRALIA

Professor Dr Yaman Ors TURKEY

Mr D B Paintin UK

Dr Mark Parascandola USA

Dr Mike Parker UK

Dr E Pellegrino USA

Dr Leo Pessini BRAZIL

Dr Neil Pickering NEW ZEALAND

Dr Emma Pickworth UK

Professor Patrick Pietroni UK

Professor Anthony Pinching UK

Professor A Pollock UK

Professor A Pompidou FRANCE

Dr Mike Porter UK

Professor Ren-Zong Qiu CHINA

Dr Bashir Qureshi UK

Dr Gillian Raab UK

Dr Janet Radcliffe-Richards UK
Professor Jennifer Radden USA

Dr Dilip Raje UK

Dr Fiona Randall UK

Prof Dr Stella Reiter-Theil SWITZERLAND

Dr Leone Risdale UK

Dr Wendy Rogers UK

Ms Gillian Romano-Critchley UK

Dr Miguel Ruiz-Canela SPAIN

Professor Hyakadai Sakamoto JAPAN

Dr Paul Salkovkis UK

Dr Jane Sandall UK

Assoc Professor Margareta Sanner SWEDEN

Dr John Saunders UK

Dr Wendy Savage UK

Professor P A Scott UK

The Revd Professor Mary Seller UK

Dr Hugh Series UK

Dr Michael Sharpe UK

Dr Aziz Sheikh UK

Dr Peter Skegg NEW ZEALAND

Professor Loane Skene AUSTRALIA

Dr Anne Slowther UK

Dr Nigel Smeeton UK

Dr Samuel Smith UK

Dr Elizabeth Snowden UK

Dr A Sommerville UK

The Revd Peter Speck UK

Dr Timothy Squires UK

Dr Marc Stauch UK

Dr Marcel Stchedroff UK

Professor Gordon Stirrat UK

Dr Danny Sullivan UK

Dr George Szmukler UK

Dr Victor Tadros UK

Dr Michael Cheng-tek Tai ROC

Dr Ian Tait UK

Dr Sandy Thomas UK

Mrs H M Thornton UK

Dr Bernadette Tobin AUSTRALIA

Dr J H Tripp UK

Dr Daniel Fu-chang Tsai ROC

Dr Gareth Tudor-Williams UK

Dr Kate Tunna UK

Dr M Tweeddale UK

Professor Duncan Vere UK

Revd Bryan Vernon UK

Dr Paul Wainwright UK

Ms Patricia Walsh UK

Dr Stellan Welin SWEDEN

Dr F O Wells UK

Dr Henry Wellsmore AUSTRALIA

Dr Dorothy Wertz USA

Professor Richard West UK

Dr Tom Wilkie UK

Dr Clare Williams UK

Professor R C N Williamson UK

Assistant Professor Josephine Wong CHINA

Professor John Wyatt UK

Professor Tatsu Yamamoto JAPAN

Dr Zbigniew Zalewski POLAND

Dr A S Zigmond UK

Dr Ron Zimmern UK

Dr Deborah Zion AUSTRALIA 